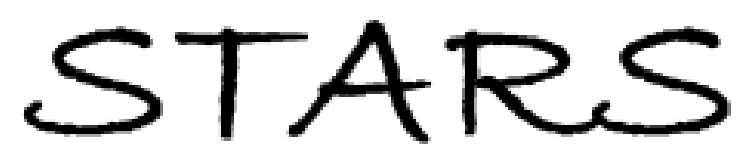

University of Central Florida

STARS

Faculty Bibliography 2010s

Faculty Bibliography

$1-1-2015$

\title{
Infrared surface polaritons on bismuth
}

\author{
Farnood Khalilzadeh-Rezaie \\ University of Central Florida \\ Christian W. Smith \\ University of Central Florida \\ Janardan Nath \\ University of Central Florida \\ Nima Nader \\ Monas Shahzad \\ University of Central Florida
}

See next page for additional authors

Find similar works at: https://stars.library.ucf.edu/facultybib2010

University of Central Florida Libraries http://library.ucf.edu

This Article is brought to you for free and open access by the Faculty Bibliography at STARS. It has been accepted for inclusion in Faculty Bibliography 2010 s by an authorized administrator of STARS. For more information, please contactSTARS@ucf.edu.

\section{Recommended Citation}

Khalilzadeh-Rezaie, Farnood; Smith, Christian W.; Nath, Janardan; Nader, Nima; Shahzad, Monas; Cleary, Justin W.; Avrutsky, Ivan; and Peale, Robert E., "Infrared surface polaritons on bismuth" (2015). Faculty Bibliography 2010s. 6622.

https://stars.library.ucf.edu/facultybib2010/6622

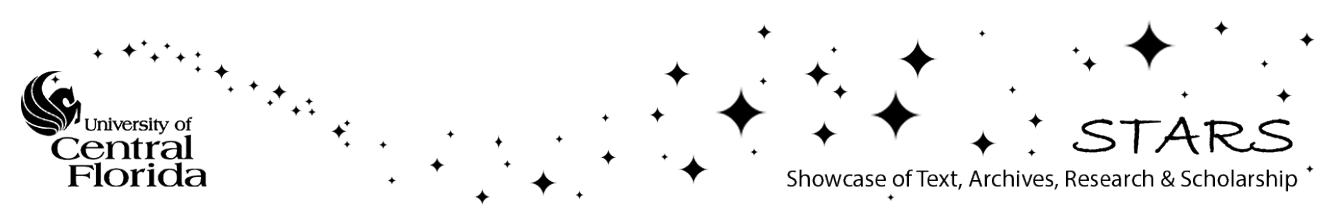




\section{Authors}

Farnood Khalilzadeh-Rezaie, Christian W. Smith, Janardan Nath, Nima Nader, Monas Shahzad, Justin W. Cleary, Ivan Avrutsky, and Robert E. Peale 


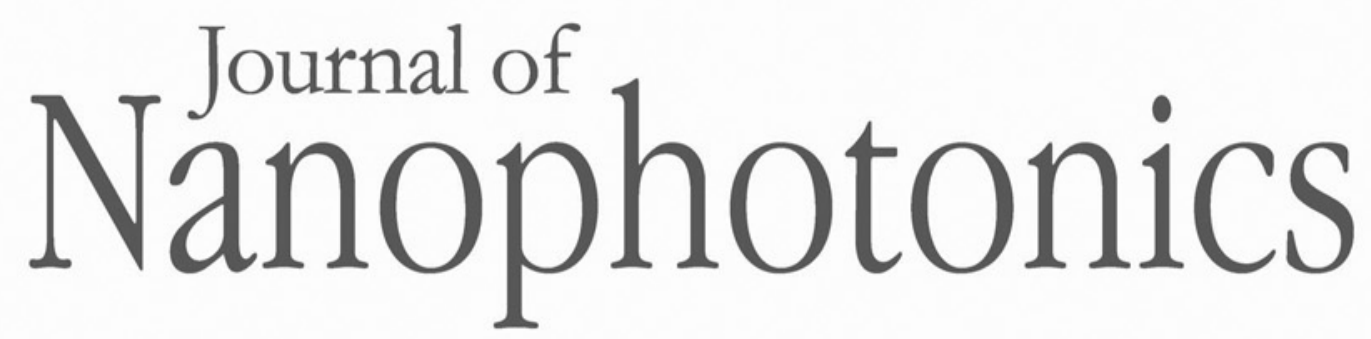

Nanophotonics.SPIEDigitalLibrary.org

\section{Infrared surface polaritons on bismuth}

Farnood Khalilzadeh-Rezaie

Christian W. Smith

Janardan Nath

Nima Nader

Monas Shahzad

Justin W. Cleary

Ivan Avrutsky

Robert E. Peale 


\title{
Infrared surface polaritons on bismuth
}

\author{
Farnood Khalilzadeh-Rezaie, ${ }^{\text {a,* }}$ Christian W. Smith, ${ }^{\text {a,b,c,d }}$ Janardan Nath, ${ }^{\text {a }}$ \\ Nima Nader, ${ }^{c, e}$ Monas Shahzad, ${ }^{\text {, }} \dagger$ Justin W. Cleary, \\ Ivan Avrutsky, ${ }^{f}$ and Robert E. Peale ${ }^{a}$ \\ ${ }^{a}$ University of Central Florida, Department of Physics, 4000 Central Florida Boulevard, Orlando, \\ Florida 32816, United States \\ bUniversity of Central Florida, Nanoscience Technology Center, 4000 Central Florida \\ Boulevard, Orlando, Florida 32816, United States \\ ${ }^{\mathrm{c}}$ Air Force Research Laboratory, Sensors Directorate, Wright-Patterson Air Force Base, \\ Ohio 45433, United States \\ ${ }^{\mathrm{d}}$ Wyle Laboratories Inc., 2601 Mission Point Boulevard, Suite 300, Dayton, Ohio 45431, \\ United States \\ ${ }^{\mathrm{e}}$ Solid State Scientific Corporation, 12 Simon Street, Nashua, New Hampshire 03060, \\ United States \\ ${ }^{f}$ Wayne State University, Department of Electrical and Computer Engineering, \\ 5050 Anthony Wayne Drive, Detroit, Michigan 48202, United States
}

\begin{abstract}
Optical constants for evaporated bismuth (Bi) films were measured by ellipsometry and compared with those published for single crystal and melt-cast polycrystalline $\mathrm{Bi}$ in the wavelength range of 1 to $40 \mu \mathrm{m}$. The bulk plasma frequency $\omega_{p}$ and high-frequency limit to the permittivity $\varepsilon_{\infty}$ were determined from the long-wave portion of the permittivity spectrum, taking previously published values for the relaxation time $\tau$ and effective mass $m^{*}$. This part of the complex permittivity spectrum was confirmed by comparing calculated and measured reflectivity spectra in the far-infrared. Properties of surface polaritons (SPs) in the long-wave infrared were calculated to evaluate the potential of Bi for applications in infrared plasmonics. Measured excitation resonances for SPs on Bi lamellar gratings agree well with calculated resonance spectra based on grating geometry and complex permittivity. (C) 2015 Society of Photo-Optical Instrumentation Engineers (SPIE) [DOI: 10.1117/1.JNP.9.093792]
\end{abstract}

Keywords: bismuth; infrared; surface polariton; grating coupler; semimetal; specular reflectance; scattering matrix analysis.

Paper 14137SS received Oct. 31, 2014; accepted for publication Dec. 29, 2014; published online Feb. 17, 2015.

\section{Introduction}

Surface plasmon polaritons (SPPs) are surface-bound electromagnetic (EM) waves described by macroscopic electrodynamics with potential applications in logic and sensing, a blossoming field known colloquially as plasmonics. ${ }^{1-5}$ SPPs have been studied extensively at visible wavelengths on good conductors and, to a lesser extent, at infrared wavelengths. Different infrared (IR) SPP host materials have been investigated, including metals, ${ }^{6,7}$ semimetals, ${ }^{8}$ metal-silicides, and semiconductors. $^{9-12}$ A review of infrared plasmonic host materials is found in Ref. 13. Properties such as dispersion, penetration depth, and propagation length are determined by the complex permittivity spectra of the conductor and adjoining dielectric. SPPs are excited by TM-polarized light (electric field in plane of incidence) using suitable coupling structures ${ }^{7,12,14}$ when the real part of a conductor's permittivity is negative and exceeds in magnitude the always-positive imaginary part. ${ }^{14-18}$

\footnotetext{
*Address all correspondence to: Farnood Khalilzadeh-Rezaie, E-mail: farnood_rezaie@knights.ucf.edu

${ }^{\dagger}$ Current address: Forman Christian College, Department of Physics, Lahore, Pakistan.
} 
This paper presents a demonstration of surface polaritons (SPs) in a material [the semimetal bismuth (Bi)] and in a frequency regime (long-wave IR), for which the real part of the permittivity is small but positive and comparable to the imaginary part. ${ }^{4,5,8}$ The surface EM wave and the surface polarization, which couple to form the SP, are physically identical to SPPs. The wave function and the field distributions are the same as for SPPs, namely those of an inhomogeneous plane wave that propagates along, and decays exponentially away from, the surface. The source of the fields is a polarization of free electrons at the surface, i.e., it is a traveling plasma oscillation. Such SPs have been already described in detail for the semimetal Sb (Ref. 8) and for heavily doped $\mathrm{Si}^{1{ }^{11}}$ Thus, the label SP is mainly used to conform to the abstract convention that SPPs should exist only when the real part of the permittivity is negative. Here we evaluate the potential of SPs on Bi for plasmonic applications.

The complex permittivity spectrum of the semimetal Bi has been measured before from visible to far-IR wavelengths, but the samples studied were oriented single crystals ${ }^{19,20}$ or melt-cast and mirror-polished polycrystalline samples. ${ }^{21}$ Evaporated Bi films are more likely to be used in applications, and an unanswered question is how the optical properties of these films compare with those for crystalline samples. To this end, we measured the permittivity spectra for electronbeam (e-beam) and thermally evaporated Bi films by ellipsometry from wavelengths of 1.4 to $40 \mu \mathrm{m}$. To validate the measured optical constants, we measured far-IR reflectivity spectra on Bi films and long-wave IR optical excitation resonances for SPs on Bi lamellar gratings. These measurements were shown to agree well with calculations based on our permittivity values.

A motivation for this study was that SPs on Bi have potential plasmonic device applications due to their tight mode confinement at IR frequencies when compared to traditional plasmon hosts, such as noble metals. Tightly bound SPs in the IR molecular fingerprint range could be used for real-time sensing of biomolecule interactions on suitably functionalized surfaces. ${ }^{22,23}$

\section{Theoretical Considerations}

The wave vector for propagation of SPs along a conductor surface ( $x$ direction) is

$$
K_{\mathrm{SP}}(\omega)=\frac{\omega}{c} \sqrt{\frac{\varepsilon_{d} \varepsilon_{c}}{\varepsilon_{d}+\varepsilon_{c}}},
$$

where $\varepsilon_{d}$ and $\varepsilon_{c}$ are complex permittivities of the dielectric and conductor, respectively. The wavevector for propagation normal to the surface ( $z$ direction) is

$$
K_{z d, z c}=\sqrt{K_{\mathrm{SPP}}-\varepsilon_{d, c}\left(\frac{\omega}{c}\right)^{2}},
$$

where subscript $d$ is for dielectric and holds in the region $z>0$, and subscript $c$ is for conductor and holds for $z<0$. When $\operatorname{Re}[\varepsilon]$ is large and negative as for good metals at visible and longer wavelengths, or when it is small and positive but comparable to or smaller than $\operatorname{Im}[\varepsilon]$ as in $\mathrm{Sb}$ (Ref. 8) in the long-wave IR, the SP decays exponentially away from, and propagates along, the interface. Bi turns out to be similar to Sb regarding the sign and relative magnitudes of the real and imaginary parts of the permittivity in the long-wave IR. This is unsurprising as both are semimetals with similar carrier concentrations.

The characteristic intensity propagation length and field penetration depth into the media bounding the interface are important to applications. The intensity propagation length is

$$
L_{x}=\frac{1}{2 \operatorname{Im}\left[K_{\mathrm{SP}}(\omega)\right]},
$$

and the field penetration depth is

$$
L_{d, c}=\left[\frac{\omega}{c} \operatorname{Im} \sqrt{\frac{\varepsilon_{d, c}^{2}}{\varepsilon_{d}+\varepsilon_{c}}}\right]^{-1} .
$$


Below the plasma frequency, $K_{\mathrm{SP}}$ exceeds the wavevector of a freely propagating EM wave of the same frequency. To excite SPs with light, a coupling structure, such as prism or grating, is required. In the IR, gratings are preferred for reasons described in Ref. 24. A grating adds or subtracts integer multiples of the fundamental grating momentum to the in-plane component of the photon momentum so that it may match the SP momentum ${ }^{6,7}$ according to

$$
\sin \theta+\frac{m \lambda}{p}= \pm \frac{c}{\omega} \operatorname{Re}\left[K_{\mathrm{SP}}\right]
$$

where $p$ is the grating period, $m$ is an integer of either sign, $\lambda$ is the wavelength of the incident EM light, and $\theta$ is the angle of incidence.

The long-wavelength permittivity $\varepsilon=\varepsilon^{\prime}+i \varepsilon^{\prime \prime}$ of a conductor can usually be described by the Drude model: ${ }^{10,19}$

$$
\varepsilon=\varepsilon_{\infty}\left[1-\frac{\left(\frac{\omega_{p}}{\omega}\right)^{2}}{1+i\left(\frac{m}{m^{*}}\right)\left(\frac{1}{\omega \tau}\right)}\right],
$$

where $\tau$ is the relaxation time and $\varepsilon_{\infty}$ is the real part of permittivity well above the plasma frequency, which can be defined here as

$$
\omega_{p}^{2}=4 \pi N e^{2} /\left(m^{*} \varepsilon_{\infty}\right) .
$$

This definition (in Gaussian units) is somewhat different than in Ref. 19. This is done so that in the case $\omega \tau \gg 1$, when $\varepsilon$ is real, the value of $\omega_{p}$ is identified by the zero-crossing of the permittivity. Then $\omega_{p}$ has the same physically intuitive interpretation as for good metals, for which $\varepsilon_{\infty}=1$ and $m=m^{*}$. In the case of Bi near the zero-crossing of $\varepsilon^{\prime}$, we actually have $\omega \tau \sim 1$, so that the zerocrossing frequency is redshifted and has the value $\sqrt{\omega_{p}^{2}-\left(m / m^{*}\right)^{2} \tau^{-2}}$. Using $m^{*} / m=0.92$ and $\tau^{-1}=270 \mathrm{~cm}^{-1}$ from Ref. 19 allows us to determine $\omega_{p}$ from the zero-crossing of $\varepsilon^{\prime}$, and we are left with the single parameter $\varepsilon_{\infty}$ to fit Eq. (6) to the $\varepsilon^{\prime}$ spectrum. For completeness, we give the equations for $\varepsilon^{\prime}$ and $\varepsilon^{\prime \prime}$ as

$$
\begin{gathered}
\varepsilon^{\prime}=\varepsilon_{\infty}\left[1-\frac{\left(\frac{\omega_{p}}{\omega}\right)^{2}}{1+\left(\frac{m}{m^{*}}\right)^{2}\left(\frac{1}{\omega \tau}\right)^{2}}\right], \\
\varepsilon^{\prime \prime}=\frac{\varepsilon_{\infty}\left(\frac{m}{m^{*}}\right)\left(\omega_{p} \tau\right)^{2}}{\left[(\omega \tau)^{2}+\left(\frac{m}{m^{*}}\right)^{2}\right] \omega \tau} .
\end{gathered}
$$

When $\omega \tau \ll 1$, the limiting form of $\varepsilon^{\prime \prime}$ goes as $1 / \omega$, as it should. ${ }^{25}$ Far-IR reflectivity spectra are calculated from the complex permittivity spectra according to Fresnel's equations, namely

$$
R=\left|\frac{1-\sqrt{\varepsilon}}{1+\sqrt{\varepsilon}}\right|^{2} .
$$

This same expression was used to obtain an $\varepsilon^{\prime}$ spectrum from experimental values of $\varepsilon^{\prime \prime}$ and $R$ presented in Ref. 19.

\section{Experimental Details}

Bi films were thermally and e-beam evaporated from $99.999 \%$ pure Bi pellets onto one-side polished (100) silicon substrates. Multiple evaporations were required to build up an optically thick layer, with each layer thickness confirmed using a Veeco Dektak step profilometer. The IR penetration depth into $\mathrm{Bi}$ was estimated from IR transmittance spectra for films of different thicknesses to be $\sim 3 \mu \mathrm{m}$ at $10 \mu \mathrm{m}$ wavelength. ${ }^{26,27}$ A thermally evaporated $\mathrm{Bi}$ film of $12 \mu \mathrm{m}$ thickness was prepared for ellipsometry measurements, and this sample was considered optically thick, such that no transmitted light reached the substrate.

An e-beam evaporated film of $6 \mu \mathrm{m}$ thickness was also studied, but even though this was twice the characteristic penetration depth, there was evidence in the ellipsometry and reflectivity 
spectra of Fabry-Perot fringes, indicating penetration to and reflection from the substrate. A study of the thickness dependence of the amplitude of these fringes was published in Ref. 26 and showed that the fringe amplitude decreases monotonically with increasing thickness, and they are no longer observable for thicknesses of 8 microns and higher.

The Bi films were characterized by a J.A. Woollam IR-VASE ellipsometer, which spans the wavelength range from 1.4 to $40 \mu \mathrm{m}$. The complex permittivity spectrum was calculated from the raw ellipsometer output using standard Fresnel equations, ${ }^{25,28}$ assuming no contribution from the substrate.

The normal-incidence far-IR reflectivity spectrum was measured using a Bomem DA8 spectrometer with reflectivity accessory inside the evacuated sample compartment. The resources used were a globar source, mylar pellical beamsplitters of thickness 3 and $6 \mu \mathrm{m}$, and a room temperature deuterated triglycine sulfate (DTGS) pyroelectric detector. The useful spectral range for these measurements was 50 to $700 \mathrm{~cm}^{-1}$ (200 to $14 \mu \mathrm{m}$ wavelength).

Lamellar gratings for SP generation experiments were fabricated by photolithography. An $\sim 1 \mu \mathrm{m}$-thick layer of photoresist was spun on an Si substrate followed by UV exposure under a grating mask with a $20 \mu \mathrm{m}$ period and $50 \%$ duty cycle. This was followed by development in a tetramethylammonium hydroxide based solution. A thick Bi coating was thermally evaporated on the gratings. The grating profile was characterized by cross-sectional scanning electron microscopy (Hitachi FE-SEM SU-70). The x-ray diffraction (XRD) pattern of Bi films was acquired by a Rigaku D/Max system in symmetric out-of-plane mode with Bragg-Brentano geometry (with $30 \mathrm{kV}$ and $40 \mathrm{~mA}, \mathrm{Cu} \mathrm{K} \alpha$ radiation at $\lambda=0.1540598 \mathrm{~nm}$ ) for $2 \theta$ values from 10 to $80 \mathrm{deg}$ in $0.05 \mathrm{deg}$ increments.

The specular reflectance of the grating was measured using a Daylight Solutions quantum cascade laser with a $0.1 \mathrm{~nm}$ line width, 7.9 to $10.5 \mu \mathrm{m}$ tuning range, $5 \mathrm{~nm}$ wavelength step size, and a motor-controlled goniometer. The laser was electronically chopped at $1 \mathrm{kHz}$, i.e., the laser was turned on and off with 50\% duty cycle. A mercury cadmium telluride detector measured the reflected intensity. A lock-in synchronously amplified the signal. The grating was mounted on the goniometer so that its rulings were orthogonal to the plane of incidence for the TM-polarized beam. A LabView program controlled the angle of incidence and source wavelength, and the program recorded the lock-in output. A reference was collected after each sample measurement by replacing the grating with a gold mirror. The reflectance was found by dividing sample and reference spectra.

\section{Results and Discussion}

As-deposited films appear shiny and mirror-like. Figure 1 presents SEM images of the surfaces of thermally and e-beam evaporated Bi films. The thermally evaporated sample is composed of nanoparticles of $\mathrm{Bi}$ with characteristic lengths of 100 to $200 \mathrm{~nm}$. The e-beam evaporated film has a larger average particle size in the range from 200 to $600 \mathrm{~nm}$. The films are smooth on the length scale of all wavelengths considered in this paper. The morphology has the appearance of overlapping platelets with facetted edges, so that the films appear to be nanocrystalline rather than amorphous. Differences in the porosity and concentration of boundaries for the two films may cause their permittivity spectra to differ from each other and from that of single crystal $\mathrm{Bi}$.

Figure 2 presents XRD $\theta-2 \theta$ measurement of both thermal and e-beam evaporated Bi films. The e-beam evaporated Bi films exhibit reflections only from (003), (006), and (009) planes, which suggests that the film is well oriented with the Bi trigonal axis perpendicular to the substrate. $^{29}$ The thermally evaporated film has additional reflections from (104) and (202), suggesting a lower degree of orientation. The broad weak peak at 69 deg is due to the substrate, as confirmed by its disappearance when the sample is tilted slightly off-normal to the plane of incidence. All of the sharp peaks belong to crystalline Bi. If the interpretation of the SEM images as being overlapping nanocrystalline platelets is correct, the larger platelets and thinner layer for the e-beam evaporated sample might give a more oriented, less jumbled, pile of particles, which would explain the difference in the XRD results.

The complex permittivity spectra of our Bi films are presented in Fig. 3. The $\varepsilon^{\prime}$ values are negative beyond $\sim 31 \mu \mathrm{m}$ wavelength, which indicates a smaller plasma frequency than for $\mathrm{Sb}$, 

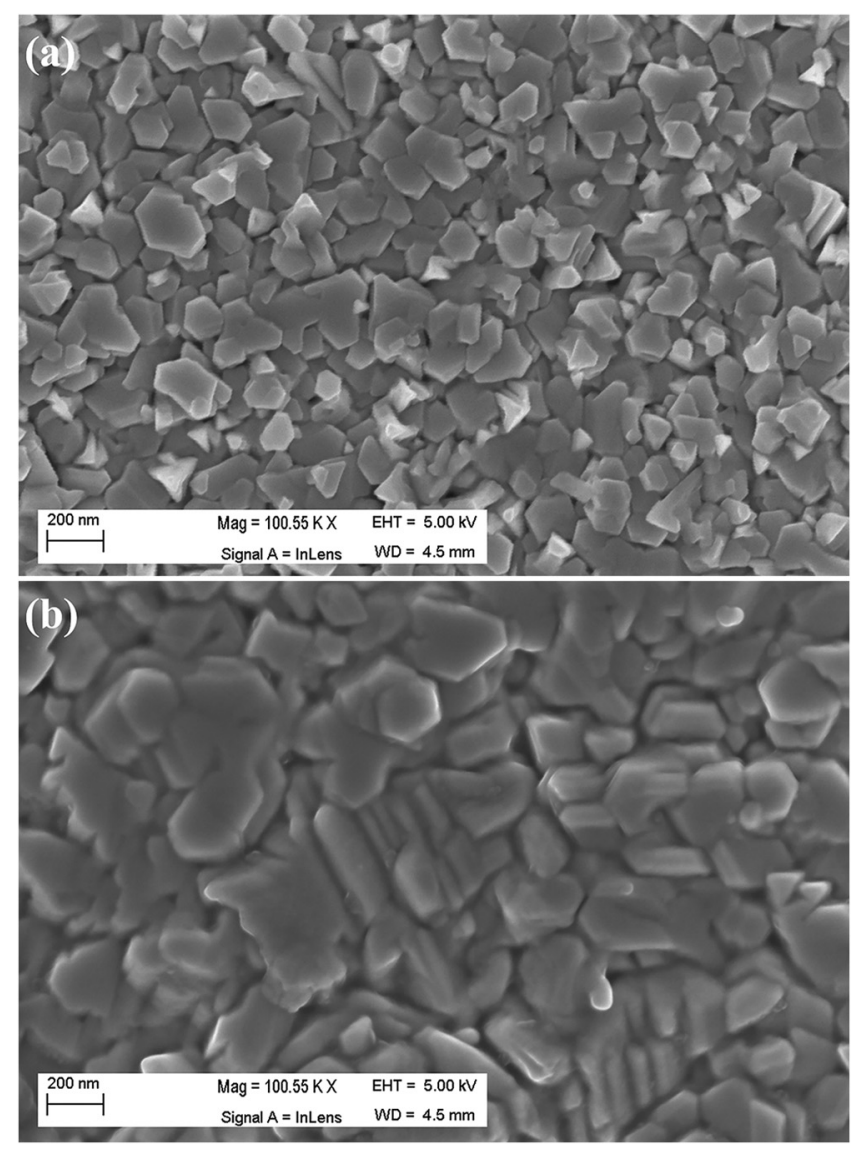

Fig. 1 Scanning electron microscopy (SEM) image of (a) thermally evaporated and (b) electronbeam (e-beam) evaporated bismuth (Bi) thin films.

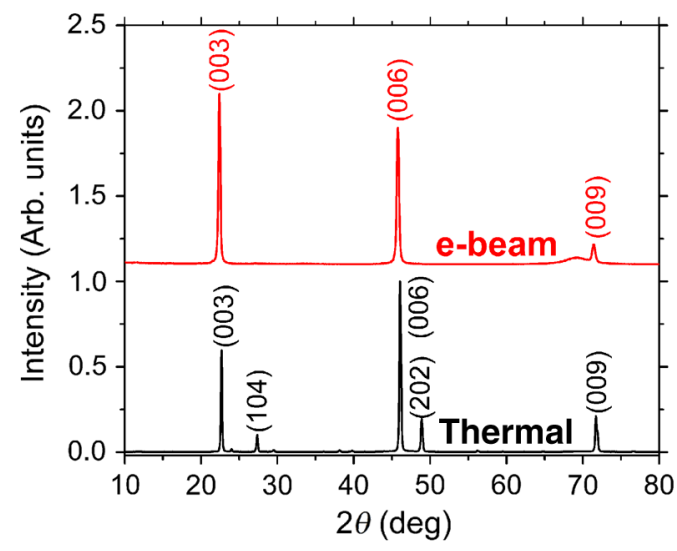

Fig. 2 X-ray diffraction of evaporated Bi films.

where the cross-over occurs at $11 \mu \mathrm{m} .{ }^{8}$ The $\varepsilon^{\prime \prime}$ values are positive over the whole spectrum, as thermodynamically required. ${ }^{25}$ In the wavelength region of our SP studies (8 to $10.5 \mu \mathrm{m}$ ), $\varepsilon^{\prime}>0$, and its value is comparable to that of $\varepsilon^{\prime \prime}$. This situation is similar to that of $\mathrm{Sb}$ in the same region, where bound SP waves were demonstrated.

Comparison is made in Fig. 3 with previous near- to long-wave IR results for melt-cast polycrystalline Bi (Ref. 21) and for oriented single crystal ${ }^{19,20} \mathrm{Bi}$. The short-wave permittivity values for the evaporated films are significantly smaller. This may be a consequence of porosity and grain boundaries in the evaporated film. The e-beam evaporated film has higher permittivity 


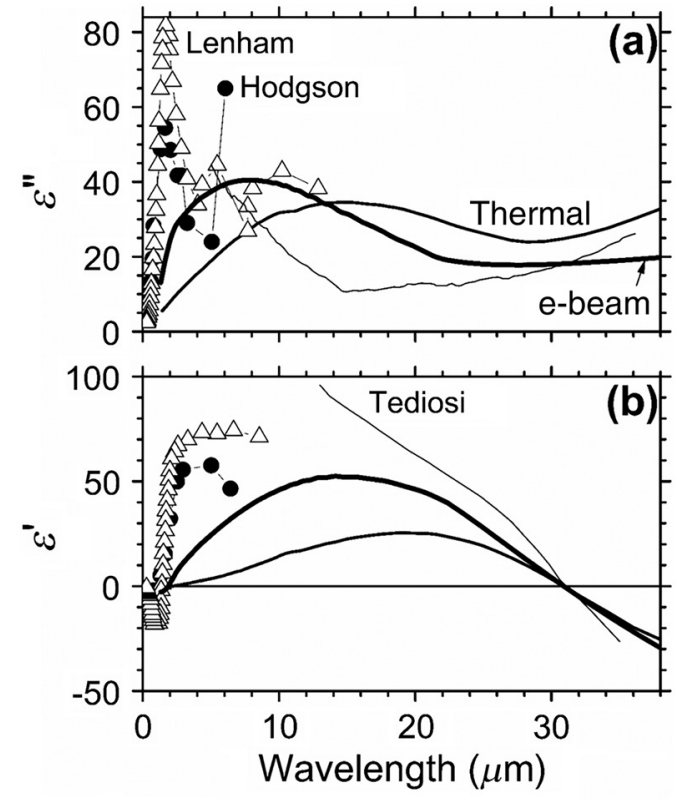

Fig. 3 Permittivity spectra for thermally and e-beam evaporated $\mathrm{Bi}$. (a) Imaginary part. (b) Real part. Comparison is made to prior reports for melt-cast polycrystalline or trigonally oriented crystalline samples.

values for shorter wavelength than does the thermally evaporated film, and this may be explained as due to its larger grains so that the film behaves more like a continuous metal.

The permittivity spectrum of Ref. 19 for single crystal Bi oriented along the trigonal axis is the only other published result for wavelengths longer than 12 microns. We calculated an $\varepsilon^{\prime}$ spectrum from their presented normal-incidence reflectivity spectrum $\mathbf{R}$ and the $\varepsilon^{\prime \prime}$ spectrum, which was itself calculated from their presented conductivity spectrum, $\varepsilon^{\prime \prime}=\sigma / \varepsilon_{0} \omega$ (S.I. units, $\varepsilon_{0}=$ vacuum permittivity). ${ }^{19}$ Significant differences in the $\varepsilon^{\prime \prime}$ values for the three curves point to loss mechanism of morphological rather than fundamental origin. The larger grained e-beam evaporated sample may be expected to behave more like a continuous film, and its $\varepsilon^{\prime \prime}$ spectrum is indeed closer to that of the single crystal result.

Using the observed zero-crossing at $30.86 \mu \mathrm{m}$ for $\varepsilon^{\prime}$, together with values for $\tau$ and $m^{*}$ from Ref. 19, determines the value $\omega_{p}=437 \mathrm{~cm}^{-1}$. Then, fitting Eq. (8) to the Fig. 3 spectrum in the range from 30 to $38 \mu \mathrm{m}$ determines the $\varepsilon_{\infty}$ value to be 112 , which is essentially the same as the value 108 given in Ref. 19. The spectrum for the thermally evaporated film and the fit are

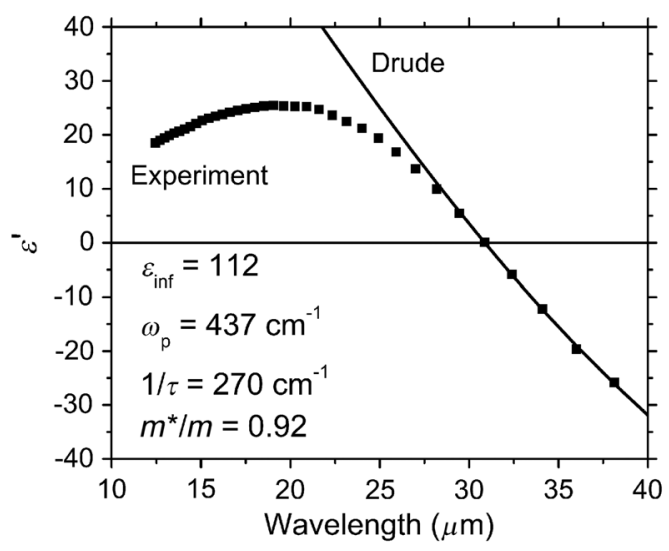

Fig. 4 Permittivity spectrum compared to Drude calculation. The real part of the permittivity for the thermally evaporated film is plotted as symbols, while the Drude calculation is given by the solid line. Drude parameters used are indicated. 


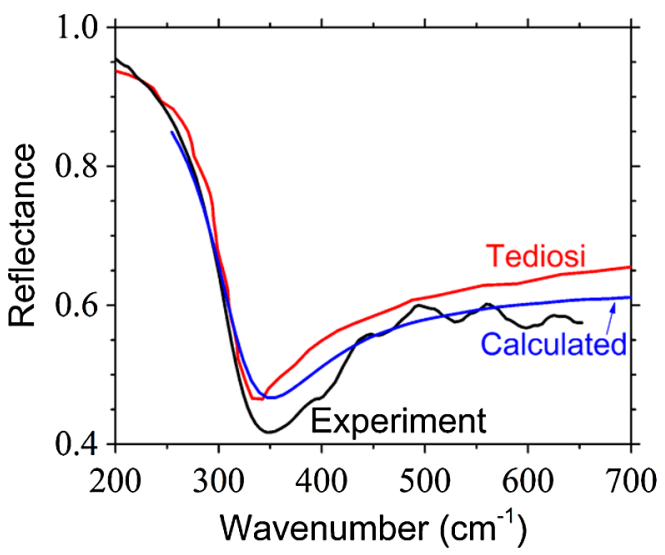

Fig. 5 Measured and calculated reflectance from an e-beam made Bi sample is compared with Ref. 19.

presented in Fig. 4, and the agreement is excellent for wavelengths of $30 \mu \mathrm{m}$ and beyond. (The spectrum for the e-beam evaporated film is very similar, see Fig. 3.). From Eq. (7), we determine the concentration of carriers to be $2.2 \times 10^{20} \mathrm{~cm}^{-3}$, which is at least $20 \times$ larger than the values previously reported for molecular beam epitaxy grown Bi films. ${ }^{30}$

Calculated $\varepsilon^{\prime \prime}$ spectra from Eq. (9) give poor agreement with experimental results in both shape and magnitude. This indicates that the main source of far-IR loss is something other than free electron absorption at these wavelengths. A possible source is multiphonon absorption, but the difference between thermal and e-beam evaporated samples shows that there are also losses of a technological, rather than fundamental, nature.

Figure 5 compares the measured reflectance for an e-beam evaporated $6-\mu$ m-thick Bi film with calculations [Eq. (10)] based on our permittivity spectrum and with prior measurement. ${ }^{19}$ The measured spectrum shows Fabry-Perot oscillations because the film thickness is only twice the skin depth and there is a large index mismatch between the $\mathrm{Bi}\left(\varepsilon^{\prime} \sim 40\right.$ to 50 , see Fig. 3$)$ and the silicon substrate $\left(\varepsilon^{\prime}=11.7\right)$ at these wavelengths. The similarity between calculated and measured $R$ spectra confirms our ellipsometer-measured permittivity spectra. The minimum of reflectance occurs at $\sim 343 \mathrm{~cm}^{-1}$, and the absorption feature is somewhat broader and deeper than reported earlier for the crystal sample. ${ }^{19}$

Calculated physical characteristics of SPs on Bi are presented next. We note that the polariton nature of the dispersion curve for $\mathrm{Bi}$ is similarly weak as for $\mathrm{Sb},{ }^{8}$ i.e., the SP dispersion curve lies close to the light line with a small deviation near the plasma frequency, in contrast to the curve for a good metal. Nevertheless, Bi supports bound surface waves with physical characteristics of SPPs. The SP propagation length $L_{x}$ and field penetration depths $L_{d, c}$, calculated from permittivity data using Eqs. (3) and (4), are presented in Fig. 6. A broad shallow dip for Bi occurs

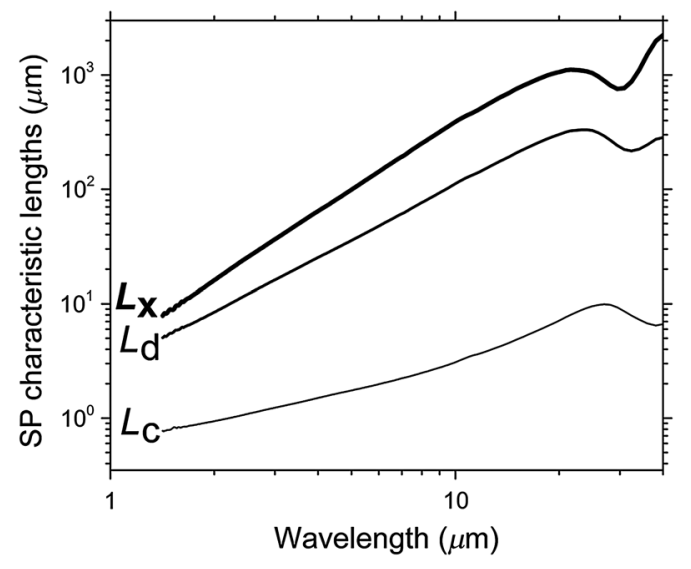

Fig. 6 Surface polariton characteristic lengths as a function of free-space wavelength. 


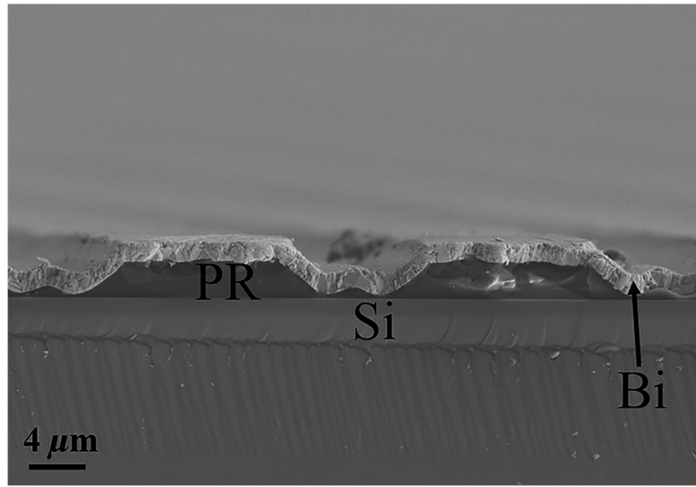

Fig. 7 SEM cross-section of Bi gratings. Si substrate, photoresist ridges (PR), and thermally evaporated $\mathrm{Bi}$ coating are indicated.

around 31 microns, where $\varepsilon^{\prime}$ changes sign. At $10 \mu \mathrm{m}$ wavelength, $L_{x}$ is $\sim 400 \mu \mathrm{m}$. Here, $L_{d} \sim 100 \mu \mathrm{m}$, which is comparable to the value for Ag at the same wavelength. ${ }^{10}$ Even at $30 \mu \mathrm{m}$ wavelength near the plasma frequency, the field penetration above $\mathrm{Bi}$ is still $200 \mu \mathrm{m}$, i.e., still not subwavelength, though this is a $5 \times$ tighter confinement than would be the case for Ag here. ${ }^{10}$ The penetration depth of the field into the conductor $L_{c}$ is $\sim 3 \mu \mathrm{m}$ at $10 \mu \mathrm{m}$ wavelength, i.e., it is about the same as the IR penetration depth, as it should be.

Figure 7 presents an SEM image of the grating cross-section. The average groove depth is $2.0 \mu \mathrm{m}$. The thickness of the thermally evaporated $\mathrm{Bi}$ is $1.4 \mu \mathrm{m}$, which is only half the penetration depth at 10 microns wavelength, so that the SP fields will penetrate to the Si and photoresist. Due to the coating of the groove side walls, the duty of the grating bars is $\sim 65 \%$, i.e., it exceeds the $50 \%$ duty cycle of the photomask.

Figure 8(a) presents measured and calculated reflectance spectrum from the Bi grating at a $64 \mathrm{deg}$ angle of incidence. A resonance due to excitation of SPs appears near $9.5 \mu \mathrm{m}$, which agrees with Eq. (5) for the $m=-4$ order. The calculated spectrum (scattering matrix method $^{31,32}$ ) that gave the best agreement (plotted) assumed a grating amplitude of $1.4 \mu \mathrm{m}$ and a grating-bar duty of $65 \%$, values somewhat smaller than, and larger, respectively, those obtained for the actual grating (Fig. 7). Calculations using our permittivity values and those

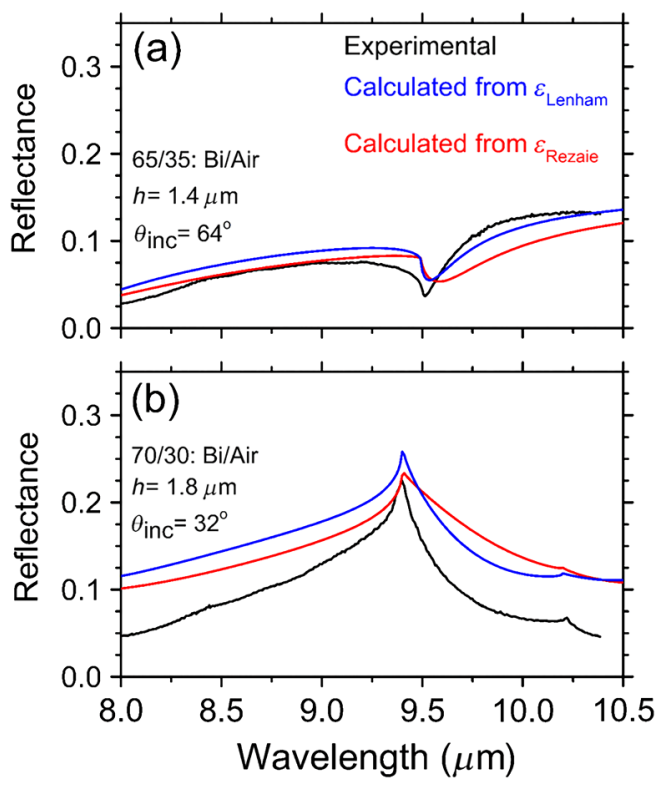

Fig. 8 Specular reflectance of $\mathrm{Bi}$ gratings at (a) 64 deg angle of incidence, $m=-4$ resonance order and (b) 32 deg angle of incidence, $m=1$ resonance order. 


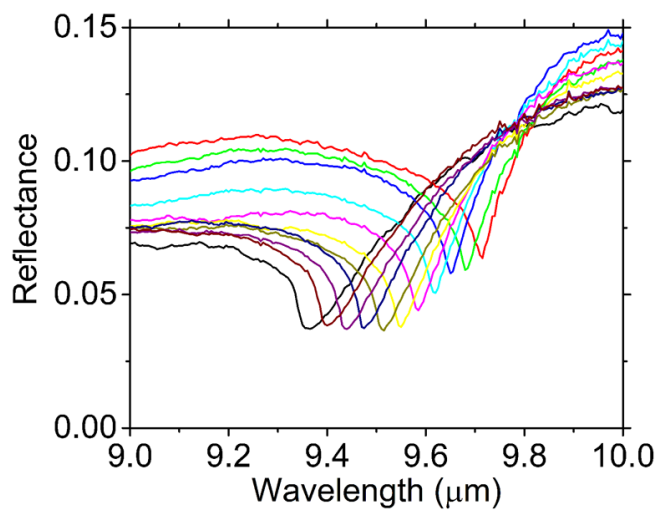

Fig. 9 Reflectance spectra for $\mathrm{Bi}$ gratings at different incidence angles corresponding to the $m=-4$ resonance. Resonances correspond to incidence angles from 60 deg to 70 deg in steps of 1 deg in order of increasing wavelength.

for crystal Bi are similar despite an approximately fivefold difference in $\varepsilon^{\prime}$ values, although the width of the resonance calculated using the published spectrum is closer to what is observed.

Figure $8(\mathrm{~b})$ presents the measured and calculated reflectance spectrum from Bi gratings at a $32 \mathrm{deg}$ of incidence. A resonance due to excitation of SPs appears near a $9.4 \mu \mathrm{m}$ wavelength, in agreement with Eq. (5) for the $m=1$ order. The calculated spectrum with best agreement assumes a grating with an amplitude of $1.8 \mu \mathrm{m}$ and a duty cycle of $70 \%$. Again, the calculation based on the published permittivity gives a resonance width in closer agreement with observation. The absorption associated with the resonance is broad, so that the peak that signifies the edge of the resonance is the most prominent feature in Fig. 8(b). The complicated asymmetric line shapes of such resonances are discussed and explained in Ref. 33.

Figure 9 presents experimental reflectance spectra for the $m=-4$ resonance at different angles of incidence. Resonances sharpen and redshift as the incidence becomes more oblique. This order's absorption is sharper and more resonance-like, and hence more interesting for sensing applications. The positions of the resonances follow the predictions of Eq. (5).

The calculated SP properties and resonances have been determined from the measured permittivity spectrum, which does not explicitly identify the nature of the polarization responsible for the SP fields. In the Introduction, we stated that this polarization was plasmonic, i.e., due to free carriers. The existence of free carriers is reasonably presumed for the semimetal $\mathrm{Bi}$, and the clearly Drude-like permittivity beyond $30 \mu \mathrm{m}$ wavelength supports this. However, in the region of our experiments, the permittivity is not Drude-like, and we note that different types of surface polarizations are also known to produce SPs, e.g., phonon polaritons ${ }^{34}$ and magnetic polaritons. ${ }^{35}$ However, these may be discounted for the nonmagnetic and homopolar $\mathrm{Bi}$, a rather heavy atom, which would have optical phonons at much longer wavelengths and without a dipole moment. Thus, a plasmon source for the SP fields seems the most reasonable conclusion.

The permittivity spectra reported by different authors, and for differently prepared evaporated films, are significantly different. These differences remain poorly understood, though they appear correlated with differences in morphology. In particular, at $2 \mu \mathrm{m}$ wavelength, the more coarse the microstructure in going from single crystal ${ }^{20}$ to melt-cast, ${ }^{21}$ to thermal, and to e-beam evaporated samples, the smaller are both the real and imaginary parts of the permittivity. We do not expect artifacts due to scattering to appear beyond visible wavelengths given the nanocrystalline size distribution in our films. In the long-wavelength region, the larger-grained ebeam evaporated sample's permittivity is closer to that of the single crystal.

In summary, this article reports measured permittivity spectra for evaporated Bi films in the range of 1.4 to 40 microns. We studied the excitation resonances for IR SPs in evaporated Bi gratings, and we found that calculated spectra based on our permittivity values agree well with the observed spectra. The IR resonances in the range of 8 to 10.5 microns are distinct and potentially useful in sensing applications. Hence, $\mathrm{Bi}$, whose plasma frequency is more than two orders smaller than traditional metal hosts, such as Au, has potential for mid- to long-wave IR plasmonic applications. 


\section{Acknowledgments}

The authors acknowledge help of Professor G. Boreman in ellipsometry measurements. Work by UCF authors was partly supported by the Florida High Technology Corridor (I-4) program. F.K.R. acknowledges support from SPIE Optics and Photonics Education Scholarship and University of Central Florida's Research Excellence Fellowship. J.W.C., N.N., and C.W.S. acknowledge support from Air Force Office of Scientific Research) under LRIR No. 12RY10COR (Program Officer Dr. Gernot Pomrenke). R.E.P. and J.W.C. conceived the experiments. F.K.R. was responsible for sample fabrication, characterization, and reflectance measurements. J.W.C. and M.S. performed the ellipsometry measurements. C.W.S. and N.N. helped with optical measurements of gratings. I.A. calculated of the reflectance spectrum using scattering matrix analysis. F.K.R., J.N., J.W.C., and R.E.P. performed data analysis and prepared the figures. F.K.R. acknowledges Dr. Andrew Warren for his assistance during $\mathrm{X}$-ray diffraction measurements. All coauthors contributed to the writing of the article.

\section{References}

1. E. Kretschmann and H. Raether, "Radiative decay of non radiative surface plasmons excited by light (surface plasma waves excitation by light and decay into photons applied to nonradiative modes)," Zeitschrift Fuer Naturforschung, Teil A 23, 2135 (1968).

2. E. Kretschmann, "Decay of non radiative surface plasmons into light on rough silver films. Comparison of experimental and theoretical results," Opt. Commun. 6(2), 185187 (1972).

3. D. Sarid and W. Challener, Modern Introduction to Surface Plasmons: Theory, Mathematica Modeling, and Applications, Cambridge University Press, Cambridge (2010).

4. F. Yang, J. Sambles, and G. Bradberry, "Long-range surface modes supported by thin films," Phys. Rev. B 44(11), 5855 (1991).

5. F. Yang, G. Bradberry, and J. Sambles, "Experimental observation of surface exciton-polaritons on Vanadium using infrared radiation," J. Mod. Opt. 37(9), 1545-1553 (1990).

6. H. Raether, Surface Plasma Oscillations and Their Applications, Academic Press, New York (1977).

7. J. W. Cleary et al., "Long-wave infrared surface plasmon grating coupler," Appl. Opt. 49(16), 3102-3110 (2010).

8. J. W. Cleary et al., "Infrared surface polaritons on antimony," Opt. Express 20(3), 26932705 (2012).

9. R. Soref, R. E. Peale, and W. Buchwald, "Longwave plasmonics on doped silicon and silicides," Opt. Express 16(9), 6507-6514 (2008).

10. J. Cleary et al., "IR permittivities for silicides and doped silicon," JOSA B 27(4), 730-734 (2010).

11. M. Shahzad et al., "Infrared surface plasmons on heavily doped silicon," J. Appl. Phys. 110(12), 123105 (2011).

12. J. C. Ginn et al., "Infrared plasmons on heavily-doped silicon," J. Appl. Phys. 110(4), 043110 (2011).

13. S. Law, V. Podolskiy, and D. Wasserman, "Towards nano-scale photonics with micro-scale photons: the opportunities and challenges of mid-infrared plasmonics," Nanophotonics 2(2), 103-130 (2013).

14. H. Raether, Surface Plasmons on Smooth Surfaces, Springer, Berlin, Heidelberg (1988).

15. P. Halevi and A. Boardman, Electromagnetic Surface Modes, Wiley, New York (1982).

16. M. G. Cottam and D. R. Tilley, Introduction to Surface and Superlattice Excitations, CRC Press, New York (2010).

17. B. E. Sernelius, Surface Modes in Physics, John Wiley \& Sons, New York (2011).

18. K. Welford, "Surface plasmon-polaritons and their uses," Opt. Quantum Electron. 23(1), 1-27 (1991).

19. R. Tediosi et al., "Charge carrier interaction with a purely electronic collective mode: plasmarons and the infrared response of elemental bismuth," Phys. Rev. Lett. 99(1), 016406 (2007). 
20. A. Lenham, D. Treherne, and R. Metcalfe, "Optical properties of antimony and bismuth crystals," JOSA 55(9), 1072-1074 (1965).

21. J. Hodgson, "The infra-red properties of bismuth," Proc. Phys. Soc. B 67(3), 269 (1954).

22. J. Homola, "Present and future of surface plasmon resonance biosensors," Anal. Bioanal. Chem. 377(3), 528-539 (2003).

23. J. Homola, S. S. Yee, and G. Gauglitz, "Surface plasmon resonance sensors: review," Sens. Actuators B Chem. 54(1), 3-15 (1999).

24. J. W. Cleary et al., "Infrared surface plasmon resonance biosensor," Proc. SPIE 7673, 767306 (2010).

25. L. D. Landau et al., Electrodynamics of Continuous Media, Elsevier, Amsterdam (1984).

26. M. Shahzad, Infrared Surface Plasmon Polaritons on Semiconductor, Semimetal and Conducting Polymer, University of Central Florida Orlando, Florida (2012).

27. M. Shahzad et al., "Infrared surface waves on semiconductor and conducting polymer," Proc. SPIE 8024, 80240B (2011).

28. H. Tompkins and E. A. Irene, Handbook of Ellipsometry, William Andrew, Norwich, New York (2005).

29. F. Yang et al., "Large magnetoresistance of electrodeposited single-crystal bismuth thin films," Science 284(5418), 1335-1337 (1999).

30. C. Hoffman et al., "Semimetal-to-semiconductor transition in bismuth thin films," Phys. Rev. B 48(15), 11431 (1993).

31. D. Whittaker and I. Culshaw, "Scattering-matrix treatment of patterned multilayer photonic structures," Phys. Rev. B 60(4), 2610 (1999).

32. M. Liscidini et al., "Scattering-matrix analysis of periodically patterned multilayers with asymmetric unit cells and birefringent media," Phys. Rev. B 77(3), 035324 (2008).

33. A. Hessel and A. Oliner, "A new theory of Wood's anomalies on optical gratings," Appl. Opt. 4(10), 1275-1297 (1965).

34. G. Borstel and H. Falge, Surface Phonon-Polaritons, pp. 221-248, Wiley, Chichester (1982).

35. M. Jensen et al., "Experimental observation of magnetic surface polaritons in Fe F 2 by attenuated total reflection," Phys. Rev. Lett. 75(20), 3756 (1995).

Farnood Khalilzadeh-Rezaie is a PhD candidate in the University of Central Florida (UCF) Department of Physics and received his BS in atomic-molecular physics from the University of Tehran. He is a graduate research assistant in the UCF physics department, winner of the SPIE Optics and Photonics Education Scholarship, a Northrop-Grumman Fellow and a UCF Graduate Research Excellence Fellow. His current research interests are in infrared plasmonics and novel applications of thin-film semiconductors in energy, sensing, and communication.

Christian W. Smith is a research assistant at the University of Central Florida. His research focuses on novel applications for thin-film semiconductors and development of plasmonic sensing devices. He received his $\mathrm{PhD}$ in physics from the University of Central Florida in 2014.

Janardan Nath is a PhD student in the Department of Physics at the University of Central Florida. He has done his master's in physics (MSc) from the Indian Institute of Technology Guwahati (IIT). His research interest includes study of metamaterial absorbers, infrared detectors, thin films technology, plasmonics materials, and chalcogenide glass.

Nima Nader is a postdoctoral researcher at the University of Arizona, College of Optical Sciences, and a contracting research scientist at the Air Force Research Labs, Sensors Directorate, WPAFB, Ohio. His primary research involves quantum optics, infrared plasmonics for quantum communication, single photon sources, detection means, and on-chip components. His current projects include investigation of coupling of plasmonic antenna to lower-dimensional quantum systems, such as quantum dots and quantum wells, development of new materials as IR plasmonic hosts, such as conductive zinc oxides and metal germanides for group IV based systems. He received his PhD in physics from the University of Central Florida in 2014. 
Monas Shahzad obtained his MSc in physics with specialization in electronics from GC University, Lahore, Pakistan, in 2001. He received his MS and PhD in physics from the University of Central Florida in 2010 and 2012, respectively, on a Fulbright Scholarship. He is an assistant professor in the Department of Physics, Forman Christian College, Lahore, Pakistan. His research interest is to study surface plasmon polariton resonances in novel materials in the form of thin film and at nano-scale.

Justin W. Cleary is a research physicist at the Air Force Research Labs, Sensors Directorate, WPAFB, Ohio. His primary research involves investigation and development of infrared plasmonics for novel detection means and on-chip components. His current projects include development of conductive zinc oxide hole arrays for extraordinary optical transmission and light trapping, and metal germanide plasmonics for group IV based systems. He received his $\mathrm{PhD}$ in physics from the University of Central Florida in 2010.

Ivan Avrutsky is associate professor in the Department of Electrical and Computer Engineering, Wayne State University. He leads the Integrated Optics and Nano-Photonics Lab, which is a member of C-PHOM, NSF Center for Photonics and Multiscale Nanomaterials. Before joining Wayne State, he was a postdoctoral fellow at the Nortel Institute for Emerging Technologies, University of Toronto, and senior research fellow at the General Physics Institute of the Russian Academy of Sciences in Moscow.

Robert E. Peale received a BA from the University of California in 1983, an MS from Cornell University in 1986, and a $\mathrm{PhD}$ from Cornell in 1990, all in physics. He was a postdoctoral research associate at Lehigh University for two years before becoming an assistant professor of physics at UCF in 1991. He holds the rank of full professor. 